\title{
Surface Modification of Fluorine Contained Resins using an Elongated Parallel Plate Electrode Type Dielectric Barrier Discharge Device
}

\author{
Masaaki Nagatsu ${ }^{1,2 *}$, Kazuya Sugiyama ${ }^{2}$, Iuliana Motrescu ${ }^{1,3}$, Mihai Alexandru \\ Ciolan $^{1}$, Akihisa Ogino ${ }^{2}$, and Naohisa Kawamura ${ }^{4}$ \\ ${ }^{1}$ Research Institute of Electronics, Shizuoka University, \\ ${ }^{2}$ Graduate School of Integrated Science and Technology, Shizuoka University, \\ 3-5-1 Johoku, Naka-ku, Hamamatsu, 432-8561, Japan \\ ${ }^{3}$ Science Department \& Research Institute for Agriculture and Environment, University \\ of Agricultural Sciences and Veterinary Medicine of Iasi, Iasi, 700490, Romania \\ ${ }^{4}$ Kumeta Manufacturing Corp., Yoshida-cho, Haibara-gun, 421-0301, Japan \\ *nagatsu.masaaki@shizuoka.ac.jp
}

In this study, we developed an elongated parallel plate type dielectric barrier discharge(DBD) device with pre-ionization electrodes for large-area surface modification of fluorine contained resin films, such as polytetrafluoroethylene (PTFE) and ethylene tetrafluoroethylene (ETFE). By optimizing a phase difference between pre-ionization and main discharge voltages, we confirmed that an intensified DBD plasma was generated between main electrodes having an electrode area of $50 \times 200 \mathrm{~mm}^{2}$ and a gap of $5 \mathrm{~mm}$. Uniformity of surface modification of fluorine contained resin films treated by DBD plasmas were examined under air and helium circumstances using carboxyl-reactive fluorescence dye for different polymer materials (PTFE, ETFE and polyethylene) and treatment times.

Keywords: Surface modification, Fluorine contained resin, Polytetrafluoroethylene, Atmospheric pressure dielectric barrier discharge, Carboxyl group

\section{Introduction}

Fluorine contained resins, such as polytetrafluoroethylene (PTFE), ethylenetetrafluoroethylene (ETFE), etc., show their excellent dielectric, thermal and mechanical properties, in addition to high chemical stability. Hence, there is a strong demand from the industry to use them in various fields, such as electronics, automotive, architectural industries, and so on. However, their poor adhesion properties with other materials limit an extent of their uses in industries, so that proper surface treatments are essential for improving their properties of adhesion with other materials.

So far, many researches have been carried out to improve the surface adhesion property of fluorine contained resin materials by using the wet chemical approaches with sodium naphthalenide treatment $[1,2]$, photochemical modification with hydrazine [3], surface modification using low pressure plasmas [4-10] and atmospheric pressure plasmas [11-15].

Among them, atmospheric pressure plasma, such as dielectric barrier discharges(DBDs) have been receiving a lot of attention because of their many advantages. DBD have been produced in different conditions and have already used for industrial applications such as ozone generation, excimer lamps, or surface treatment of different materials [16-20].

In this study, we develop a large-area atmospheric dielectric barrier discharge (DBD) plasmas for lowtemperature surface modification technique of fluorine contained resin materials. We tested three polymers, PTFE $\left(-\left(\mathrm{CF}_{2}-\mathrm{CF}_{2}\right)_{\mathrm{n}^{-}}\right)$, ETFE $\left(\left(-\mathrm{CH}_{2}-\right.\right.$ $\left.\left.\mathrm{CH}_{2}-\right)_{\mathrm{n}}-\left(-\mathrm{CF}_{2}-\mathrm{CF}_{2}-\right)_{\mathrm{m}}\right)$ and polyethylene $\left.\left(\mathrm{PE},-\left(\mathrm{CH}_{2}-\mathrm{CH}_{2}\right)_{\mathrm{n}}-\right)\right)$ to study the surface modification with carboxyl groups using the proposed large-area DBD plasmas. 


\section{Experimental}

The elongated DBD device consists of two plane parallel aluminum electrodes of $50 \times 200 \mathrm{~mm}^{2}$ with a gap of $5 \mathrm{~mm}$. The pre-ionization electrodes consist of four copper wires placed inside quartz tubes with an inner diameter of $2 \mathrm{~mm}$ and outer diameter of $4 \mathrm{~mm}$ and embedded in the lower aluminum electrode. The pre-ionization electrodes are grounded, while high voltage is applied to the lower electrode to ignite the pre-ionization discharge uniformly along the quartz surface of the pre-ionization electrodes [21]. Both the preionization and main discharge are driven by high voltage (HV) squared or sinusoidal waves at frequencies between 1 and $3 \mathrm{kHz}$. The main $\mathrm{HV}$ signal is applied between the top electrode and grounded pre-ionization $\mathrm{Cu}$ wire electrodes. As a dielectric material between two main aluminum electrodes, a polytetrafluoroethylene (PTFE) sheet with $1 \mathrm{~mm}$ thickness was placed on the lower electrode. The driving signals are supplied by a dual signal generator (NF, WF-1948), and subsequently amplified using $\mathrm{AC} / \mathrm{DC}$ amplifiers (NF, HVA 4321) up to $10 \mathrm{kV}$ peak-to-peak at the maximum. The waveforms of pre-ionization and main discharge voltages are monitored by highvoltage probes (Tektronix, P6015A) as well as the discharge current waveforms by current monitors (Pearson, model 4100) using a digital storage oscilloscope (Tektronix, DPO 4104B-L). The discharge was imaged with a high speed ICCD (Princeton Instruments, PI-MAX 3) in different circumstances.

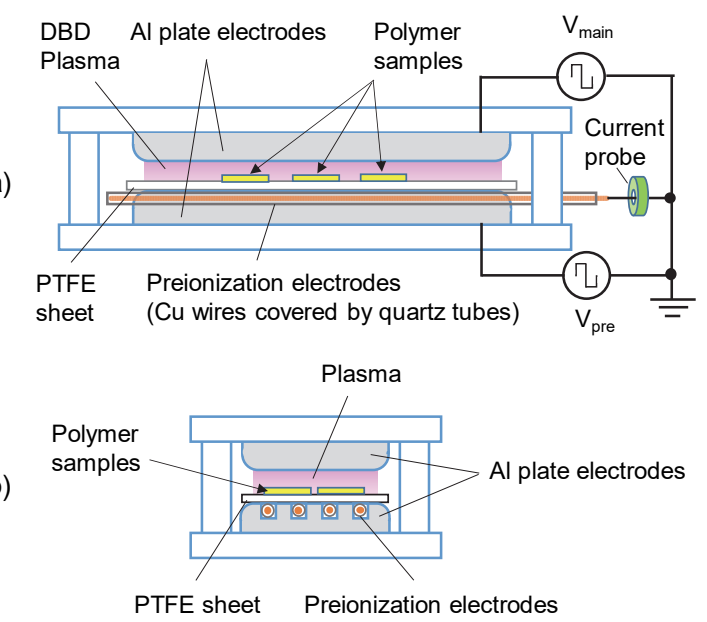

Fig. 1. Schematic drawing of the experimental setup for surface modification of polymer samples.

The visualization of the plasma surface modification was done by the chemical derivatization method using specific fluorescence dyes and fluorescence microscopy. Here, we used the fluorescent dye, Alexa Fluor ${ }^{\circledR} 594$ cadaverine (Thermo Fisher Scientific), which reacts with the carboxyl moieties on the surface of the polymer, and emits red fluorescence at $612 \mathrm{~nm}$ when excited by $599 \mathrm{~nm}$ light. The images of the functionalized areas were measured by fluorescence microscope (Leica, DMI 4000). Water contact angle of the treated samples were also analyzed.

\section{Results and discussion}

\subsection{Current-voltage characteristics of DBDs}

A typical current-voltage characteristic of the pre-ionization discharge in the experimental setup given in Fig. 1 is shown in Fig. 2, together with a top-view photo of pre-ionization discharges. Here, a frequency of applied squared waves is $1 \mathrm{kHz}$ and duty ratio is $50 \%$. It is seen that during the rising and falling phases of voltage waveform, small spiky dielectric barrier discharge currents are observed, as shown in Fig. 2 (a). The DBD plasma generated by pre-ionization discharges is clearly seen along 4 quartz tubes of electrodes as shown in Fig. 2 (b).

(a)

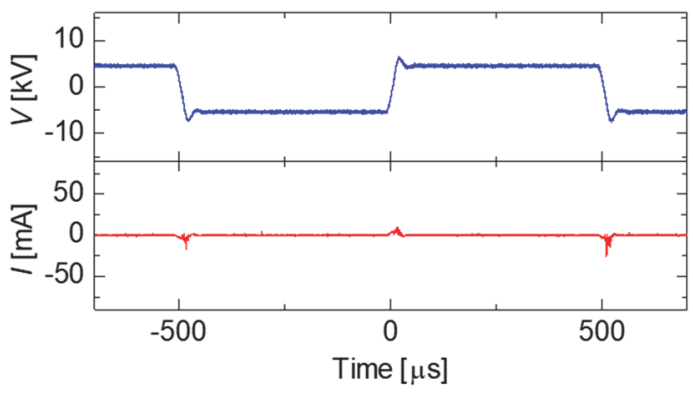

(b)

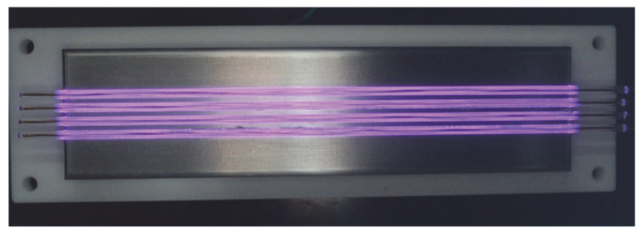

Fig. 2. (a) Current-voltage waveforms of the preionization discharge and (b) photo of pre-ionization discharges.

To realize uniform main discharges between two aluminum plate electrodes, it is essential to generate the pre-ionized discharges uniformly over the surface of bottom electrode and apply the main discharge high voltages having the same frequency as the pre-ionization voltages. The phase difference between the pre-ionization and the main discharge voltages influences the net electric field thus the breakdown of the gas. Hence, when both signals are in phase (Fig. 3 (a)), there is no main discharge, where the measured current comes only from the 
pre-ionization discharge. On the other hand, changing the phase shift between the signals to outof-phase, the electric field increases and the main discharge strongly ignites over the surface of electrodes. The current amplitude of the discharge becomes much higher as seen in the characteristics shown in Fig. 3 (b) for the case when the preionization and main signals are tuned to 180 degrees.

(a)

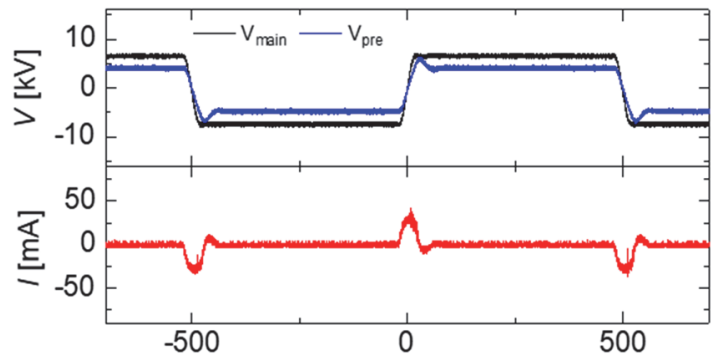

(b)

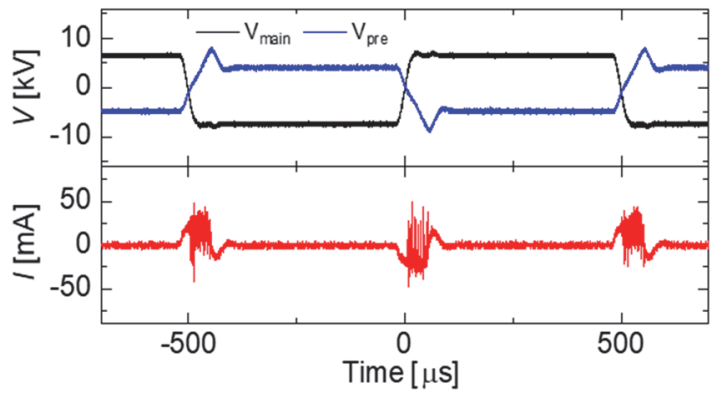

Fig. 3. Current-voltage waveforms when the preionization and main discharge voltages are (a) in-phase and (b) out-of-phase, respectively.

In atmospheric air, the onset pre-ionization discharge voltage for squared or sinusoidal wave at $1 \mathrm{kHz}$ is about $7.5 \mathrm{kVp}$-p. Keeping the preionization conditions constant at $9 \mathrm{kVp}-\mathrm{p}$, when increasing the voltage of the main discharge, for some threshold voltage at around $8 \mathrm{kVp}-\mathrm{p}$ (Fig. 4 (a)), filamentation-like discharges randomly appear in the gap between the electrodes as a result of charge accumulation at the surface of the dielectric (Fig. 4 (b)). The density of filamentation-like discharges increase with the value of the applied main voltage, and the distribution becomes relatively uniform over the surface, filling the gap between the electrodes, as shown in Fig. 4 (c). Here, these images are obtained for a gate period of 100 $\mu$ s of ICCD camera.

It is well known that $\mathrm{He}$ gas was often used to achieve uniform glow discharges at atmospheric pressure. Here, we tested the discharge under $\mathrm{He}$ gas background. Figure 5 shows typical photos of DBD plasmas taken under air (Fig. 5(a)) and He gas background (Fig. 5(b)). It is clearly seen that the DBD plasmas are more uniform in the case of $\mathrm{He}$ (a)

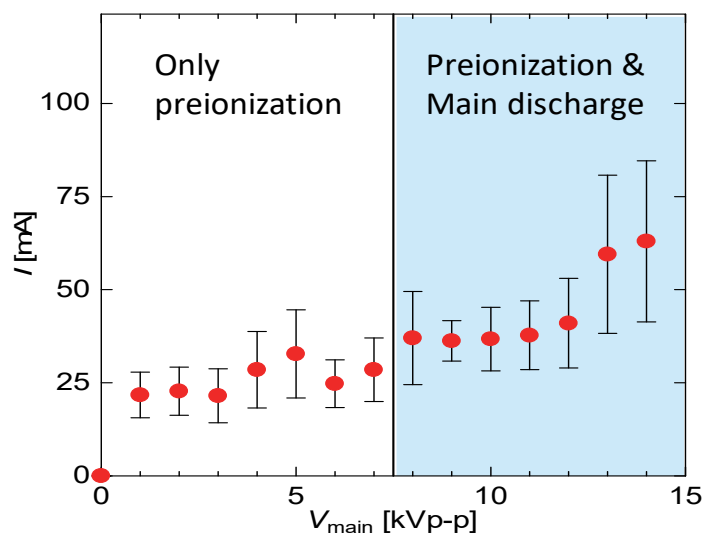

(b)

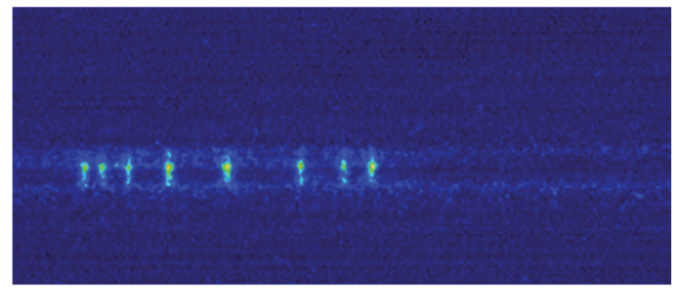

(c)

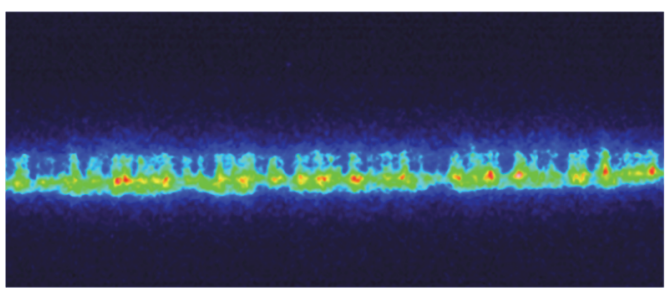

Fig. 4. (a) Relation between discharge currents and main discharge voltages at a fixed pre-ionization voltage of 9 $\mathrm{kVp}-\mathrm{p}$. ICCD images at (b) $8 \mathrm{kVp}-\mathrm{p}$ main discharge voltages and (c) $14 \mathrm{kVp}-\mathrm{p}$ main discharge voltages at a fixed pre-ionization voltage of $9 \mathrm{kVp}-\mathrm{p}$, respectively.

(a)

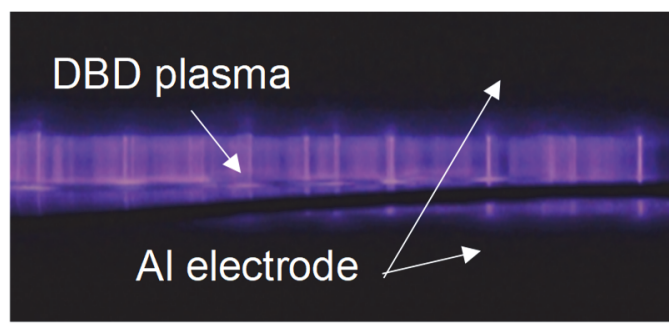

(b)

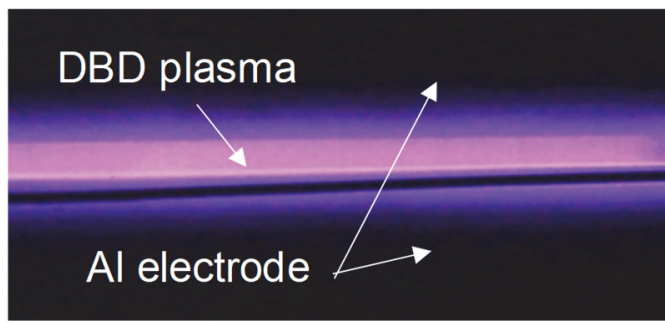

Fig. 5. Typical photos of DBD discharges at $9 \mathrm{kVp}-\mathrm{p}$ preionization and $14 \mathrm{kVp}-\mathrm{p}$ main discharge voltage under (a) air and (b) He gas background. 
gas background rather than in the case of air.

3.2. Comparison of plasma surface functionalization of PTFE, ETFE and PE under air and $\mathrm{He}$ gas atmospheres

Here we tested plasma surface functionalization onto PTFE, ETFE and PE samples by the present large-area DBD plasmas under air and $\mathrm{He}$ gas atmospheres. To compare the surface functionalization onto these sample surfaces, we performed the water contact angle measurement and chemical derivatization analysis using fluorescent dye, Alexa Fluor ${ }^{\circledR} 594$ cadaverine.

(a)

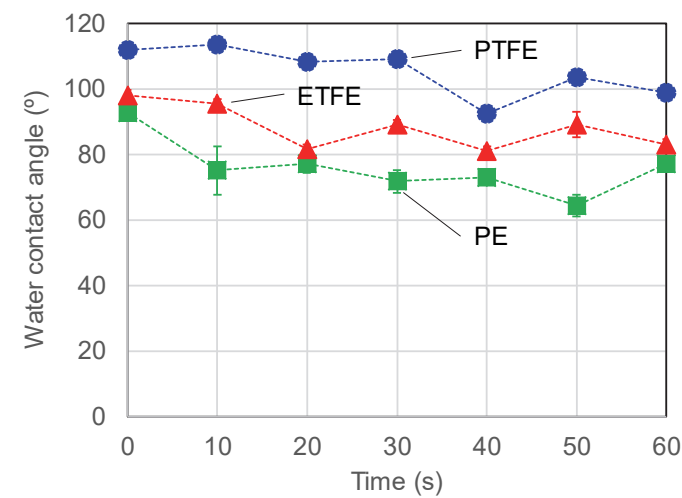

(b)

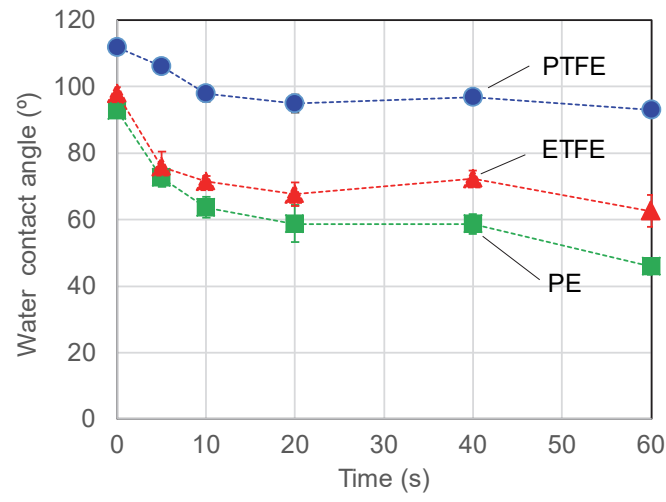

Fig. 6. Results of water contact angle measurements of PTFE, ETFE, and PE by the DBD plasma treatments under (a) air and (b) He gas.

Figure 6 shows the results of water contact angle measurements of PTFE, ETFE, and PE treated by the large-area DBD plasmas under air and $\mathrm{He}$ gas background. To examine the short-period behaviors of surface functionality, we tested the DBD treatments up to only $60 \mathrm{~s}$ duration. Under the air, the water contact angles did not change so much in $60 \mathrm{~s}$ plasma treatments for three samples. However, in the case of He gas background, they decreased from 112 to $93^{\circ}$ in PTFE, 98 to $63^{\circ}$ in ETFE, and 93 to $46^{\circ}$ in PE after $60 \mathrm{~s}$ plasma treatment, respectively. As shown in Fig. 5, plasma modification is more uniform in the case of $\mathrm{He}$ gas background compared to the air atmosphere. Hence, it is considered that surface modification was more efficient in the case of $\mathrm{He}$ gas background due to more uniform plasma irradiation over the polymer surface than the case in air.

(a)
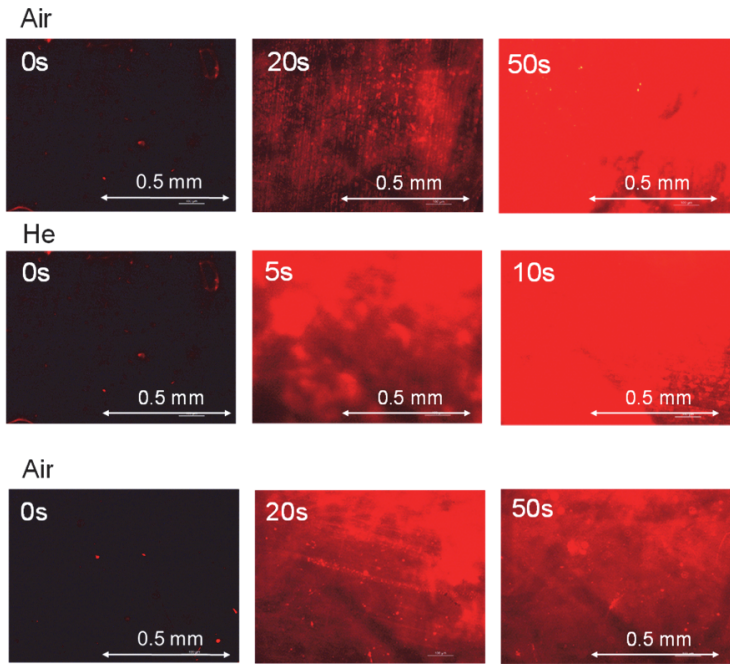

(b)
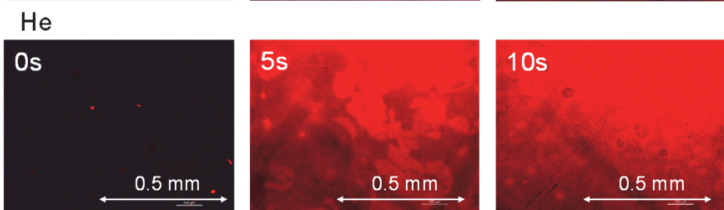

(c)
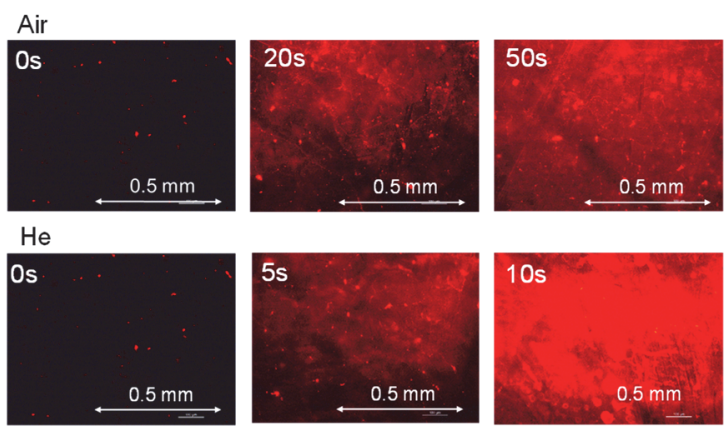

Fig. 7. Fluorescence images of (a) PTFE, (b) ETFE, and (c) PE surface for different treatment times of DBD plasmas under air (upper) and He gas background (lower).

Furthermore, the water contact angles in ETFE and PE are decreased compare to those of PTFE, and $\mathrm{PE}$ shows the largest drop in water contact angle. These results suggest that the $-\mathrm{CH}_{2}$ bonds in $\mathrm{PE}$ or ETFE are much easier to cut the bonds to form hydrophilic functional groups than $-\mathrm{CF}_{2}$ bonds in ETFE or PTFE. From Fig. 5, the largest change of water contact angle was PE, followed in order by ETFE and PTFE.

Next, we investigated the spatial uniformity of plasma functionalization over the surfaces of PTFE, ETFE and PE samples treated by the DBD plasmas under air and He gas. Figure 7 shows the images of fluorescence microscope for different samples, 
different plasma treatments times and different background gas conditions. Figure 7 clearly shows the surface modification becomes more uniform after longer plasma treatment for both the cases of air and He background gas. Moreover, uniformity of surface modification in He gas background was obtained much faster than the cases in air. For the cases of air, the images show the spot-like patterns of fluorescence dyes even at $50 \mathrm{~s}$. This might be due to the filamentation-like plasma discharges as shown in Fig. 5. On the other hand, the fluorescence images are more uniform in the case of He gas even in a short treatment time of $10 \mathrm{~s}$. These results suggest that rapid and uniform plasma modification could be achieved in $\mathrm{He}$ gas background. Finally, it is noted that even in PTFE, we can modify the surface very easily similar to the cases of ETFE and PE.

\section{Conclusion}

In this study, we developed an elongated parallel plate electrode type DBD plasma device with preionization electrodes for large-area surface modification of fluorine contained resin films, such as PTFE and ETFE, in addition to non-fluorine PE film. We presented the development of elongated parallel plate electrode type DBD plasma device. By tuning a phase difference between pre-ionization and main discharge voltages to out-of-phase, we confirmed that an intensified DBD plasma was generated between main electrodes having an electrode area of $50 \times 200 \mathrm{~mm}^{2}$ and a gap of $5 \mathrm{~mm}$. Here, the pre-ionization is very important to achieve the uniform DBD plasmas over the electrode surface at lower ignition voltage of main discharges. Surface modification of fluorine contained resin films treated by DBD plasmas under air and helium circumstances were examined using water contact angle measurements and carboxyl-reactive fluorescence dye for different polymer materials (PTFE, ETFE and PE) and treatment times.

\section{Acknowledgements}

This work was supported in part by Grant-in-Aid for Scientific Research (No. 17H02804) from the Japan Society for the Promotion of Science (JSPS). The authors would like to express their thanks to Kumeta Manufacturing Co. Ltd. for collaborative research and Mr. Orita of Shizuoka University for helping the present experiment.

\section{References}

1. K. Ha, S. McClain, S. L. Suib, and A. Garton, J. Adhes., 33 (1991) 169.

2. L. M. Siperko and R. R. Thomas, J. Adhes. Sci. Technol., 3 (1989) 157.

3. U. Meyer, S. Kostler, V. Ribitsch, and W. Kern, Macromol. Chem. Phys., 206 (2005) 210.

4. E. T. Kang, K. L. Tan, K. Kato, Y. Uyama, and Y. Ikada, Macromolecules, 29 (1996) 6872.

5. J. P. badey, E. Espuche, D. Sage, B. Chabert, Y. Jugnet, C. Batier, and T. M. Duc, Polymer, 17 (1996) 1377.

6. D. J. Wilson, R. L. Williams, and R. C. Pond, Surf. Interface Anal., 31 (2001) 385.

7. T. K. Markkula, J. A. Hunt, F. R. Pu, and R. L. Williams, Surf. Interface Anal., 34 (2002) 583.

8. N. Vandencasteele, D. Merche, and F. Reniers, Surf. Interface Anal., 38 (2006) 526.

9. T.-K. Lin, S.-J. Wu, C.-K. Peng, and C.-H. Yeh, Polym. Int., 58 (2009) 46.

10. W. Hai, T. Hi, K. Shimizu, and T. Yajima, $J$. Photopolym. Sci. Technol., 28 (2015) 479.

11. C. Z. Liu, J. Q. Wu, L. Q. Ren, J. Tong, J. Q. Li, N. Cui, N. M. D. Brown, and B. J. Meenan, Mater. Chem. Phys., 85 (2004) 340.

12. Z. Fang, L. Hao, H. Yang, X. Xie, Y. Qiu, and K. Edmund, Appl. Surf. Sci., 255 (2009) 7279.

13. J. H. Noh, H. K. Baik, I. Noh, J.-C. Park, and I.S. Lee, Surf. Coat. Technol., 201 (2007) 5097.

14. M. Nagatsu, M. Kimpara, R. Hu, and T. Abuzairi, J. Photopolym. Sci. Technol., 30 (2017) 307.

15. Y. Ohkubo, K. Ishihara, H. Sato, M. Shibahara, A. Nagatani, K. Honda, K. Endo, and Y. Yamamura, RSC Adv., 7 (2017) 6432.

16. A. Chebbah, S. Hadejeri, S. Nemmich, K. Nassour, N. Zouzou, and A. Tilmatine, OzoneSci. Eng., (2017) 1.

17. A. Abdelaziz, T. Ishijima, T. Seto, N. Osawa, H. Wedaa, and Y. Otani, Plasma Sources Sci. Technol., 25 (2016) 035012 (12 pp).

18. G. Borcia, C. A. Anderson, and N. M. Brown, Plasma Sources Sci. Technol., 12 (2003) 335.

19. B. Eliasson and U. Kogelschatz, IEEE Trans. Plasma Sci., 19 (1991) 309.

20. H. Ayan, D. Staack, G. Fridman, A. Gustol, Y. Mukhin, A. Strikovskii, A.Fridman, and G. Friedman, J. Phys. D: Appl. Phys., 42 (2009) 125202 (5 pp).

21. Y.-L. Pan, A. Bernhardt, and J. Simpson, Rev. Sci. Instrum., 43 (1972) 662. 